Proyecciones Journal of Mathematics Vol. 31, No 2, pp. 91-101, June 2012.

Universidad Católica del Norte

Antofagasta - Chile

\title{
Asymptotically convex Banach spaces and The Index of Rotundity Problem
}

\author{
FRANCISCO J. GARCÍA - PACHECO \\ UNIVERSITY OF CADIZ, THE EUROPEAN UNION \\ Received : December 2011. Accepted : January 2012
}

\begin{abstract}
The Index of Rotundity Problem asks whether a Banach space which admits equivalent renormings with index of rotundity as small as desired also admits an equivalent rotund renorming. In this paper we continue the ongoing search for a negative answer to this question by making use of a new concept: asymptotically convex Banach spaces. Some applications to The Approximation Hyperplane Series Property are given.
\end{abstract}

Subjclass : [2000] Primary 46B20, 46B10.

Keywords : Rotund, renorming, Banach space. 


\section{Introduction}

Since the concept of rotund Banach space appeared many characterizations of it have been provided. This concept motivated, among other things, the creation of an index to measure it, the so called index of rotundity.

Definition 1.1. Let $X$ be a real or complex Banach space. The index of rotundity of $X$ is defined as

$$
\zeta_{X}:=\sup \left\{\operatorname{diam}(C): C \subset S_{X} \text { is convex }\right\}
$$

The reader can quickly observe that a normed space $X$ is rotund if and only if $\zeta_{X}=0$. In [3, Theorem 7.12] it is shown an example of a Banach space which is not rotund under any equivalent renorming. Such example triggered the search for a Banach space not isomorphic to a rotund space but which can be equivalently renormed to decrease its index of rotundity as much as desired. This is the birth of the "Index of Rotundity Problem", a well-known problem among the Banach space geometers.

Question 1.2 (The Index of Rotundity Problem). Let $X$ be a real or complex Banach space. Assume that for every $h>0$ there exists an equivalent renorming $\|\cdot\|_{h}$ on $X$ such that $\zeta_{\left(X,\|\cdot\|_{h}\right)} \leq h$. Does then there exist an equivalent norm $\|\cdot\|_{0}$ on $X$ such that $\zeta_{\left(X,\|\cdot\|_{0}\right)}=0$ ?

We refer the reader to [3] for a wide perspective on rotund renormings and the index of rotundity. In the upcoming sections we continue the ongoing search for a negative answer to this question. In the last section we provide some applications to The Approximation Hyperplane Series Property.

\section{Asymptotically convex Banach spaces}

This section is devoted to describe the class of Banach spaces we will be working with.

Definition 2.1. Let $X$ be a real or complex Banach space. Consider a closed subspace $Y$ of $X$ and two numbers $\varepsilon \in(0,1]$ and $\delta \in[0,1)$. We say that $X$ is $(\varepsilon, \delta)$-asymptotically convex in the direction of $Y$ if every non-trivial segment $\left[x_{1}, x_{2}\right] \subset S_{X} \backslash S_{Y}$ verifies that dist $\left(\left[x_{1}, x_{2}\right], Y\right) \geq \varepsilon$ and $\operatorname{dist}\left(x_{1}-x_{2}, Y\right) \leq \delta\left\|x_{1}-x_{2}\right\|$. 
Examples of Banach spaces asymptotically convex follow.

Example 2.2. Take $X$ to be $\mathbf{R}^{2}$ endowed with the norm whose unit ball is

$$
B_{\ell_{\infty}^{2}} \cup B_{\ell_{2}^{2}}((-1,0), 1) \cup B_{\ell_{2}^{2}}((1,0), 1) .
$$

In this situation, $X$ is $(1,0)$-asymptotically convex in the direction of

$$
Y:=\left\{(x, y) \in \mathbf{R}^{2}: y=0\right\} .
$$

Example 2.2 can be used as a base to construct infinite dimensional asymptotically convex Banach spaces. This construction will actually show us that every Banach space is isomorphic to an asymptotically convex Banach space.

The norm of the space $X$ in Example 2.2 can actually be described as: $\|(x, y)\|=\left\{\begin{aligned} \frac{x^{2}+y^{2}}{2|x|} & \text { if }|y|<|x|, \\ |y| & \text { if }|x| \leq|y|,\end{aligned} \quad\right.$ where $x, y \in \mathbf{R}$.

Theorem 2.3. Let $Y$ and $Z$ be Banach spaces such that $Z$ is rotund. The vector space $X:=Y \times Z$ endowed with the norm

$$
\|(y, z)\|:=\left\{\begin{aligned}
\frac{\|y\|^{2}+\|z\|^{2}}{2\|y\|} & \text { if }\|z\|<\|y\|, \\
\|z\| & \text { if }\|y\| \leq\|z\|,
\end{aligned}\right.
$$

where $y \in Y$ and $z \in Z$, is a Banach space $(1,0)$-asymptotically convex in the direction of $Y \times\{0\}$.

Proof. Let $\left[\left(y_{1}, z_{1}\right),\left(y_{2}, z_{2}\right)\right]$ be a non-trivial segment contained in $S_{X} \backslash S_{Y \times\{0\}}$. Observe that the three points $\left(\left\|y_{1}\right\|,\left\|z_{1}\right\|\right),\left(\left\|y_{2}\right\|,\left\|z_{2}\right\|\right)$, and $\left(\left\|\frac{y_{1}+y_{2}}{2}\right\|,\left\|\frac{z_{1}+z_{2}}{2}\right\|\right)$ are in the unit sphere of the 2-dimensional Banach space constructed in Example 2.2. More precisely, the three previous points must be in the positive part of that unit sphere. On the other hand, notice also that $\left\|\frac{y_{1}+y_{2}}{2}\right\| \leq \frac{\left\|y_{1}\right\|+\left\|y_{2}\right\|}{2}$ and $\left\|\frac{z_{1}+z_{2}}{2}\right\| \leq \frac{\left\|z_{1}\right\|+\left\|z_{2}\right\|}{2}$, so the point $\left(\left\|\frac{y_{1}+y_{2}}{2}\right\|,\left\|\frac{z_{1}+z_{2}}{2}\right\|\right)$ is below and at the left of the point $\left(\frac{\left\|y_{1}\right\|+\left\|y_{2}\right\|}{2}, \frac{\left\|z_{1}\right\|+\left\|z_{2}\right\|}{2}\right)=$ $\frac{1}{2}\left(\left\|y_{1}\right\|,\left\|z_{1}\right\|\right)+\frac{1}{2}\left(\left\|y_{2}\right\|,\left\|z_{2}\right\|\right)$. Therefore, the only possibility is that $\left\|z_{1}\right\|=$ $\left\|z_{2}\right\|$. If $z_{1} \neq z_{2}$, then $\left\|\frac{z_{1}+z_{2}}{2}\right\|<\frac{\left\|z_{1}\right\|+\left\|z_{2}\right\|}{2}$ due to the fact that $Z$ is rotund. But this contradicts the fact that $\left(\left\|\frac{y_{1}+y_{2}}{2}\right\|,\left\|\frac{z_{1}+z_{2}}{2}\right\|\right)$ is in the unit sphere of the 2-dimensional Banach space constructed in Example 2.2. Thus $z_{1}=z_{2}$. Finally,

$$
\operatorname{dist}\left(\left[\left(y_{1}, z_{1}\right),\left(y_{2}, z_{2}\right)\right], Y \times\{0\}\right)=1
$$


and

$$
\operatorname{dist}\left(\left(y_{1}, z_{1}\right)-\left(y_{2}, z_{2}\right), Y \times\{0\}\right)=0 \text {. }
$$

Corollary 2.4. Let $X$ be a real or complex Banach space. Then $X$ can be equivalently renormed to become $(1,0)$-asymptotically convex in the direction of a closed subspace.

Proof. Consider $Z$ to be any complemented subspace of $X$ which admits an equivalent rotund renorming (for instance, any finite dimensional subspace). Assume that $Z$ is already endowed with that equivalent rotund renorming. Let $Y$ be the topological complement of $Z$ in $X$. Finally consider the norm given by Equation (2.1) on $Y \times Z$.

\section{Decreasing the index of rotundity}

The next two results are aimed at showing that a certain type of asymptotically convex Banach space can be renormed in order to decrease the index of rotundity as much as desired.

Theorem 3.1. Let $X$ be a real or complex Banach space $(\varepsilon, \delta)$-asymptotically convex in the direction of a closed subspace $Y$. Consider the equivalent norm $|\cdot|$ on $X$ given by

$$
|x|:=\sqrt{\|x\|^{2}+\operatorname{dist}^{2}(x, Y)},
$$

for all $x \in X$. Then:

1. $\zeta_{(X,|\cdot|)} \leq \max \left\{\zeta_{Y}, \sqrt{\frac{1+\delta^{2}}{1+\varepsilon^{2}}} \zeta_{X}\right\}$.

2. If $\sqrt{2} \delta<1$, then $(X,|\cdot|)$ is $(\varepsilon, \sqrt{2} \delta)$-asymptotically convex in the direction of $(Y,|\cdot|)$.

Proof. Let $x_{1}, x_{2} \in X$ such that

$$
\left|x_{1}\right|=\left|x_{2}\right|=\left|\frac{x_{1}+x_{2}}{2}\right|=1 .
$$


Notice that

$$
\begin{aligned}
4 & =\left|x_{1}+x_{2}\right|^{2} \\
& =\left\|x_{1}+x_{2}\right\|^{2}+\operatorname{dist}^{2}\left(x_{1}+x_{2}, Y\right) \\
& \leq\left\|x_{1}\right\|^{2}+\left\|x_{2}\right\|^{2}+2\left\|x_{1}\right\|\left\|x_{2}\right\| \\
& +\operatorname{dist}^{2}\left(x_{1}, Y\right)+\operatorname{dist}^{2}\left(x_{2}, Y\right)+2 \operatorname{dist}\left(x_{1}, Y\right) \operatorname{dist}\left(x_{2}, Y\right) \\
& \leq 2+2 \sqrt{\left\|x_{1}\right\|^{2}+\operatorname{dist}^{2}\left(x_{1}, Y\right)} \sqrt{\left\|x_{2}\right\|^{2}+\operatorname{dist}^{2}\left(x_{2}, Y\right)} \\
& =4 .
\end{aligned}
$$

Therefore, the previous two inequalities must indeed be equalities. In other words:

- It must happen that

$$
\left\|x_{1}+x_{2}\right\|=\left\|x_{1}\right\|+\left\|x_{2}\right\|,
$$

which means that

$$
\left[\frac{x_{1}}{\left\|x_{1}\right\|}, \frac{x_{2}}{\left\|x_{2}\right\|}\right] \subset S_{X} .
$$

- It must also be verified that

$$
\begin{aligned}
& \left\|x_{1}\right\|\left\|x_{2}\right\|+\operatorname{dist}\left(x_{1}, Y\right) \operatorname{dist}\left(x_{2}, Y\right) \\
= & \sqrt{\left\|x_{1}\right\|^{2}+\operatorname{dist}^{2}\left(x_{1}, Y\right)} \sqrt{\left\|x_{2}\right\|^{2}+\operatorname{dist}^{2}\left(x_{2}, Y\right)},
\end{aligned}
$$

which means that there exists $\alpha>0$ such that $\left\|x_{1}\right\|=\alpha\left\|x_{2}\right\|$ and $\operatorname{dist}\left(x_{1}, Y\right)=\alpha \operatorname{dist}\left(x_{2}, Y\right)$. Observe that

$$
\begin{aligned}
1 & =\left\|x_{1}\right\|^{2}+\operatorname{dist}^{2}\left(x_{1}, Y\right) \\
& =\alpha\left(\left\|x_{2}\right\|^{2}+\operatorname{dist}^{2}\left(x_{2}, Y\right)\right) \\
& =\alpha^{2} .
\end{aligned}
$$

We have now all the necessary tools to prove the assertions (1) and (2) of the theorem:

1. Let $\left[x_{1}, x_{2}\right]$ be a non-trivial segment in $S_{(X,|\cdot|)}$ :

1. Assume that $\left[x_{1}, x_{2}\right] \cap Y \neq$. Without any loss we may consider that $x_{1} \in Y$.

Then $\operatorname{dist}\left(x_{2}, Y\right)=\operatorname{dist}\left(x_{1}, Y\right)=0$, so $x_{2} \in Y$. Then $\left[x_{1}, x_{2}\right] \subset$ $S_{Y}$ and $\left|x_{1}-x_{2}\right|=\left\|x_{1}-x_{2}\right\| \leq \zeta_{Y}$. 
2. Suppose now that $\left[x_{1}, x_{2}\right] \cap Y=$. Then $\left\|x_{1}\right\|=\left\|x_{2}\right\|$ and therefore $\left[\frac{x_{1}}{\left\|x_{1}\right\|}, \frac{x_{2}}{\left\|x_{2}\right\|}\right] \subset S_{X} \backslash S_{Y}$. In the first place

$$
\left\|x_{1}\right\|=\sqrt{1-\operatorname{dist}^{2}\left(x_{1}, Y\right)}=\sqrt{1-\left\|x_{1}\right\|^{2} \operatorname{dist}^{2}\left(\frac{x_{1}}{\left\|x_{1}\right\|}, Y\right)},
$$

that is,

$$
\left\|x_{1}\right\|^{2}=\frac{1}{1+\operatorname{dist}^{2}\left(\frac{x_{1}}{\left\|x_{1}\right\|}, Y\right)},
$$

which implies that

$$
\frac{1}{\sqrt{2}} \leq\left\|x_{1}\right\| \leq \frac{1}{\sqrt{1+\varepsilon^{2}}}
$$

In the second place

$$
\begin{aligned}
\left|x_{1}-x_{2}\right| & =\sqrt{\left\|x_{1}-x_{2}\right\|^{2}+\operatorname{dist}^{2}\left(x_{1}-x_{2}, Y\right)} \\
& =\left\|x_{1}\right\| \sqrt{\left\|\frac{x_{1}}{\left\|x_{1}\right\|}-\frac{x_{2}}{\left\|x_{2}\right\|}\right\|^{2}+\operatorname{dist}^{2}\left(\frac{x_{1}}{\left\|x_{1}\right\|}-\frac{x_{2}}{\left\|x_{2}\right\|}, Y\right)} \\
& \leq\left\|x_{1}\right\| \sqrt{\left\|\frac{x_{1}}{\left\|x_{1}\right\|}-\frac{x_{2}}{\left\|x_{2}\right\|}\right\|^{2}+\delta^{2}\left\|\frac{x_{1}}{\left\|x_{1}\right\|}-\frac{x_{2}}{\left\|x_{2}\right\|}\right\|^{2}} \\
& \leq \sqrt{\frac{1+\delta^{2}}{1+\varepsilon^{2}}} \zeta_{X} .
\end{aligned}
$$

Therefore

$$
\zeta_{(X,|\cdot|)} \leq \max \left\{\zeta_{Y}, \sqrt{\frac{1+\delta^{2}}{1+\varepsilon^{2}}} \zeta_{X}\right\}
$$

2. Let $\left[x_{1}, x_{2}\right]$ be a non-trivial segment in $S_{(X,|\cdot|)} \backslash S_{(Y,|\cdot|)}$ :

1. Fix $t \in[0,1]$ and $y \in Y$. Note that $\left\|x_{1}\right\|=\left\|x_{2}\right\|=\left\|t x_{1}+(1-t) x_{2}\right\|$ and $\left[\frac{x_{1}}{\left\|x_{1}\right\|}, \frac{x_{2}}{\left\|x_{2}\right\|}\right] \subset S_{X} \backslash S_{Y}$. Thus

$$
\begin{aligned}
& \left|t x_{1}+(1-t) x_{2}-y\right| \\
= & \sqrt{\left\|t x_{1}+(1-t) x_{2}-y\right\|^{2}+\operatorname{dist}^{2}\left(t x_{1}+(1-t) x_{2}-y, Y\right)} \\
= & \left\|x_{1}\right\| \sqrt{\left\|t \frac{x_{1}}{\left\|x_{1}\right\|}+(1-t) \frac{x_{2}}{\left\|x_{2}\right\|}-\frac{y}{\left\|x_{1}\right\|}\right\|^{2}+d i s t^{2}\left(t \frac{x_{1}}{\left\|x_{1}\right\|}+(1-t) \frac{x_{2}}{\left\|x_{2}\right\|}, Y\right)}
\end{aligned}
$$




$$
\begin{aligned}
& \geq\left\|x_{1}\right\| \sqrt{2} \operatorname{dist}\left(\left[\frac{x_{1}}{\left\|x_{1}\right\|}, \frac{x_{2}}{\left\|x_{2}\right\|}\right], Y\right) \\
& \geq \varepsilon .
\end{aligned}
$$

Therefore dist $_{|\cdot|}\left(\left[x_{1}, x_{2}\right], Y\right) \geq \varepsilon$.

2. Fix a sequence $\left(y_{n}\right) \subset Y$ such that $\left(\left\|\frac{x_{1}}{\left\|x_{1}\right\|}-\frac{x_{2}}{\left\|x_{2}\right\|}-y_{n}\right\|\right)_{n \in \mathbf{N}}$ converges to $\operatorname{dist}\left(\frac{x_{1}}{\left\|x_{1}\right\|}-\frac{x_{2}}{\left\|x_{2}\right\|}, Y\right)$. For all $n \in \mathbf{N}$ :

$$
\begin{aligned}
\left|x_{1}-x_{2}-y_{n}\right| & =\sqrt{\left\|x_{1}-x_{2}-y_{n}\right\|^{2}+\operatorname{dist}^{2}\left(x_{1}-x_{2}-y_{n}, Y\right)} \\
& =\left\|x_{1}\right\| \sqrt{\left\|\frac{x_{1}}{\left\|x_{1}\right\|}-\frac{x_{2}}{\left\|x_{2}\right\|}-y_{n}\right\|^{2}+\operatorname{dist}^{2}\left(\frac{x_{1}}{\left\|x_{1}\right\|}-\frac{x_{2}}{\left\|x_{2}\right\|}, Y\right)} .
\end{aligned}
$$

As a consequence (and taking into consideration that $\left\|x_{1}\right\|=$ $\left.\left\|x_{2}\right\|\right)$

$$
\begin{aligned}
\text { dist }_{|\cdot|}\left(x_{1}-x_{2}, Y\right) & \leq\left\|x_{1}\right\| \sqrt{\delta^{2}\left\|\frac{x_{1}}{\left\|x_{1}\right\|}-\frac{x_{2}}{\left\|x_{2}\right\|}\right\|^{2}+\delta^{2}\left\|\frac{x_{1}}{\left\|x_{1}\right\|}-\frac{x_{2}}{\left\|x_{2}\right\|}\right\|^{2}} \\
& =\sqrt{2} \delta\left\|x_{1}-x_{2}\right\| \\
& \leq \sqrt{2} \delta\left|x_{1}-x_{2}\right| .
\end{aligned}
$$

Corollary 3.2. Let $X$ be a real or complex Banach space. Assume that there exist $0<\varepsilon \leq 1$ and a rotund closed subspace $Y$ of $X$ such that $X$ is $(\varepsilon, 0)$-asymptotically convex in the direction of $Y$. For every $h \in(0,2]$ there exists an equivalent norm $|\cdot|_{h}$ on $X$ such that

$$
\left.\zeta_{(X,|\cdot|}\right) \leq h .
$$

Proof. Denote by $|\cdot|_{0}$ to the original norm on $X$. For every $n \in \mathbf{N}$ we define the equivalent norm $|\cdot|_{n}$ on $X$ given by

$$
|x|_{n}:=\sqrt{|x|_{n-1}^{2}+d i s t_{|\cdot|_{n-1}}^{2}(x, Y)},
$$

for all $x \in X$.

By applying Theorem 3.1 inductively, we deduce that

$$
\zeta_{\left(X,|\cdot|_{n}\right)} \leq\left(1+\varepsilon^{2}\right)^{-\frac{n}{2}} \zeta_{X}
$$


for all $n \in \mathbf{N}$. Since

$$
\left(\left(1+\varepsilon^{2}\right)^{-\frac{n}{2}}\right)_{n \in \mathbf{N}}
$$

converges to 0 , we can find $m \in \mathbf{N}$ large enough so that

$$
\zeta_{\left(X,|\cdot|_{m}\right)} \leq h .
$$

Finally take $|\cdot|_{h}:=|\cdot|_{m}$.

We will conclude this section by showing the existence of Banach spaces with index of rotundity 2 under any equivalent renorming.

Let $\omega_{1}$ denote the first uncountable ordinal. The space of all bounded real-valued functions on $\left[0, \omega_{1}\right]$ will be denoted by $\ell_{\infty}\left(0, \omega_{1}\right)$, which becomes a Banach space endowed with the sup norm. The subspace of $\ell_{\infty}\left(0, \omega_{1}\right)$ composed of those functions with countable support is denoted by $m_{0}$. In accordance to [3, Theorem 7.12], $m_{0}$ endowed with any (non-necessarily equivalent) norm has a subspace which is linearly isometric to $m_{0}$ endowed with the sup norm. The reader may notice that the index of rotundity of $m_{0}$ endowed with the sup norm is 2 ; therefore the index of rotundity of $m_{0}$ endowed with any norm is also 2 . As a consequence, if $\Gamma$ is any uncountable set, then $\ell_{\infty}(\Gamma)$ has index of rotundity 2 under any equivalent renorming.

\section{Applications to The Approximate Hyperplane Series Prop- erty}

In [1] the authors find that the Property $\beta$ of Lindenstrauss (see [4]) is a sufficient condition for a certain pair of Banach spaces to enjoy the BishopPhelps-Bollobás Property.

Theorem 4.1 (Acosta, Aron, Garcia, and Maestre, 2008). Let $Y$ be a real or complex Banach space. If $Y$ satisfies Property $\beta$, then the pair $(X, Y)$ has the Bishop-Phelps-Bollobás Property for every Banach space $X$.

In an attempt to provide a characterization of the Bishop-Phelps-Bollobás Property, the authors of [1] came up with a new property called The Approximation Hyperplane Series Property, which we will introduce afterwards. The characterization they gave was the following:

Theorem 4.2 (Acosta, Aron, García, and Maestre, 2008). Let $Y$ be a real or complex Banach space. The following conditions are equivalent: 
1. The pair $\left(\ell_{1}, Y\right)$ has the Bishop-Phelps-Bollobás Property.

2. $Y$ satisfies the Approximation Hyperplane Series Property.

We will proceed now to define the Approximation Hyperplane Series Property. Within the next lines we will let $\mathcal{M}$ denote the set of all functions $\gamma:[0,2] \rightarrow\left[0, \frac{1}{2}\right]$ such that:

- $\lim _{\varepsilon \rightarrow 0^{+}} \gamma(\varepsilon)=\gamma(0)=0$.

- $\gamma(\varepsilon)>0$ for all $0<\varepsilon \leq 2$.

Given a real or complex Banach space $X, \gamma \in \mathcal{M}$, and $\varepsilon \in[0,2]$, we define the set $\mathcal{H S}(X, \gamma, \varepsilon)$ in the following way:

- If $\varepsilon \in(0,2]$, then $\mathcal{H} \mathcal{S}(X, \gamma, \varepsilon)$ is composed of all pairs $\left(\left(x_{k}\right)_{k \in \mathbf{N}},\left(\alpha_{k}\right)_{k \in \mathbf{N}}\right)$ such that $\left(x_{k}\right)_{k \in \mathbf{N}} \subset S_{X}, \sum_{k=1}^{\infty} \alpha_{k}$ is a convex series, and for all $A \subseteq$ $\mathbf{N}$ with $\sum_{k \in A} \alpha_{k}>1-\gamma(\varepsilon)$ and for all $\left(z_{k}\right)_{k \in A}$ with $c o\left\{z_{k}: k \in A\right\} \subset$ $S_{X}$, there exists $k_{0} \in A$ verifying that $\left\|z_{k_{0}}-x_{k_{0}}\right\| \geq \varepsilon$.

- If $\varepsilon=0$, then $\mathcal{H S}(X, \gamma, \varepsilon)$ is composed of all pairs $\left(\left(x_{k}\right)_{k \in \mathbf{N}},\left(\alpha_{k}\right)_{k \in \mathbf{N}}\right)$ such that $\left(x_{k}\right)_{k \in \mathbf{N}} \subset S_{X}$ and $\sum_{k=1}^{\infty} \alpha_{k}$ is a convex series.

Definition 4.3 (Acosta, Aron, García, Maestre, 2008). Let $X$ be a real or complex Banach space:

1. The Modulus of Hyperplane Series Approximation of $X$ with respect to $\gamma \in \mathcal{M}$ is the function

$$
\begin{aligned}
& \eta_{X}(\cdot, \gamma):[0,2] \rightarrow[0,1] \\
& \varepsilon \mapsto \eta_{X}(\varepsilon, \gamma):=\inf \left\{1-\left\|\sum_{k=1}^{\infty} \alpha_{k} x_{k}\right\|:\left(\left(x_{k}\right)_{k \in \mathbf{N}},\left(\alpha_{k}\right)_{k \in \mathbf{N}}\right) \in \mathcal{H S}(X, \gamma, \varepsilon)\right\}
\end{aligned}
$$

2. $X$ has the Approximation Hyperplane Series Property if there exists $\gamma_{X} \in$ $\mathcal{M}$ such that $\eta_{X}\left(\varepsilon, \gamma_{X}\right)>0$ for all $0<\varepsilon \leq 2$.

The geometrical interpretation of the Approximation Hyperplane Series Property is that somehow a convex series of a sequence in the unit sphere can be approximated by a unit ball supporting hyperplane. The reader may notice that the previous definition is not exactly the way the authors of [1] defined the Approximation Hyperplane Series Property. Actually, the modulus of hyperplane series approximation was later introduced in a different manuscript (see [2]). In [2] the authors prove the following result. 
Theorem 4.4 (Acosta, Aron, García-Pacheco, 2010). Let $X$ be a real or complex Banach space. If $\zeta_{X} \leq \frac{2}{3}$, then $\eta_{X}\left(\frac{\varepsilon}{3}, \gamma\right) \leq \delta_{X}(\varepsilon)$ for all $\varepsilon \in\left[3 \zeta_{X}, 2\right]$ and all $\gamma \in \mathcal{M}$.

We remind the reader that $\delta_{X}$ denotes the modulus of convexity (or modulus of Clarkson) of the Banach space $X$. Therefore, Theorem 4.4 shows that the modulus of convexity and the modulus of hyperplane series approximation are somehow related. Another result proved in [2] follows.

Theorem 4.5 (Acosta, Aron, García-Pacheco, 2010). Let $X$ be a real or complex Banach space. If $X$ admits an equivalent rotund renorming, then $X$ admits an equivalent rotund renorming failing the Approximation Hyperplane Series Property.

We will finish this section and the paper by providing a version of the previous result for asymptotically convex Banach spaces, making use of Theorem 4.4.

Theorem 4.6. Let $X$ be a real or complex Banach space. Assume that there exist $0<\varepsilon \leq 1$ and a closed subspace $Y$ of $X$ such that $X$ is $(\varepsilon, 0)$ asymptotically convex in the direction of $Y$. If there exists $\varepsilon_{0} \in\left[3 \zeta_{Y}, 2\right]$ such that $\delta_{Y}\left(\varepsilon_{0}\right)=0$, then there exists an equivalent norm on $X$ so that $X$ is $(\varepsilon, 0)$-asymptotically convex in the direction of $Y$ but lacks the Approximation Hyperplane Series Property.

Proof. In the first place, following a similar argument as in the proof of Corollary 3.2, there exists an equivalent norm $|\cdot|$ on $X$ which coincides with the original norm on $Y$ and such that

$$
\zeta_{(X,|\cdot|)} \leq \max \left\{\zeta_{Y}, \frac{\varepsilon_{0}}{3}\right\}
$$

This norm also verifies that $\varepsilon_{0} \in\left[3 \zeta_{(X,|\cdot|)}, 2\right]$; therefore by Theorem 4.4

$$
\eta_{(X,|\cdot|)}\left(\frac{\varepsilon_{0}}{3}, \gamma\right) \leq \delta_{(X,|\cdot|)}\left(\varepsilon_{0}\right)
$$

holds for all $\gamma \in \mathcal{M}$. Since $\delta_{Y}\left(\varepsilon_{0}\right)=0$, there are two sequences $\left(x_{n}\right)_{n \in \mathbf{N}},\left(y_{n}\right)_{n \in \mathbf{N}} \subset S_{Y}$ such that $\left\|x_{n}-y_{n}\right\| \geq \varepsilon_{0}$ for all $n \in \mathbf{N}$ and $\left\|\frac{x_{n}+y_{n}}{2}\right\| \rightarrow 1$ as $n \rightarrow \infty$. On the other hand, since the norm $|\cdot|$ coincides with the original norm on $Y$, we have that the two sequences 
$\left(x_{n}\right)_{n \in \mathbf{N}},\left(y_{n}\right)_{n \in \mathbf{N}} \subset S_{Y}=S_{(Y,|\cdot|)}$ are so that $\left|x_{n}-y_{n}\right|=\left\|x_{n}-y_{n}\right\| \geq \varepsilon_{0}$ for all $n \in \mathbf{N}$ and $\left|\frac{x_{n}+y_{n}}{2}\right|=\left\|\frac{x_{n}+y_{n}}{2}\right\| \rightarrow 1$ as $n \rightarrow \infty$. Therefore, $\delta_{(X,|\cdot|)}\left(\varepsilon_{0}\right)=0$ which means that $\eta_{(X,|\cdot|)}\left(\frac{\varepsilon_{0}}{3}, \gamma\right)=0$ by virtue of Equation $(4.1)$.

\section{References}

[1] M.D. Acosta, R.M. Aron, D. García, and M. Maestre, The BishopPhelps-Bollobás Theorem for operators, J. Funct. Anal. 254 11, pp. 2780-2799, (2008).

[2] M.D. Acosta, R.M. Aron, F.J. García-Pacheco, Something rare is going on with the Approximation Hyperplane Series Property, Preprint.

[3] R. Deville, G. Godefroy and V. Zizler, Smoothness and renormings in Banach spaces, Pitman Monographs and Surveys in Pure and Applied Mathematics 64, Longman Scientific, New York, (1993).

[4] J. Lindenstrauss, On operators which attain their norms, Israel J. Math. 1, pp. 139-148, (1963).

Francisco J. García-Pacheco

Department of Mathematics,

University of Cadiz,

Puerto Real (ESP) 11510,

The European Union

e-mail : garcia.pacheco@uca.es 